\section{Look at biological systems through an engineer's eyes}

SIR - Your Connections series of Essays has taken some interesting looks at the interdisciplinary study of complex, dynamic systems (see www.nature.com/nature/focus/ arts/essays/index.html). However, it has not featured a discussion of the physiological tradition of biological research, in which biological systems are analysed using reduced descriptions in much the same sense that an engineer uses a reduced description of an amplifier. An engineer is often not interested (to first order) in what is inside the box that produces gain, but studies the properties of the gain, its linearity, its frequency dependence and so on. A complete description of the structure of the amplifier is far less useful than a reduced description of its input-output relation, when the goal is to use the amplifier or connect it to other devices to make a system.

An engineer told that an unknown black box is an amplifier is rather like a biologist confronting an unknown biological system. Some structural knowledge is indispensable. Engineers would have a terrible time if they did not know which leads were power supplies, which inputs and which outputs. But the last thing an engineer would want to know is the complete circuit diagram, let alone the locations of all molecules or atoms in its resistors, capacitors and transistors. Successful investigation requires some (indispensable) knowledge of structure; but it requires many more measurements of inputs and outputs, under many conditions. Successful investigation also requires a good quantitative model of the system, called a device equation.

Physiologists have successfully analysed a large range of biological systems using this 'device-oriented' approach. For more than a century, medical students have used it to learn that the kidneys filter blood to make urine; the lungs transport oxygen from air to blood; muscles contract; sodium channels produce action potentials; and so on. Each device description in physiology — on each length scale from organ, to tissue, to cell, to organelle, to protein molecule - is associated with a device equation, just as a device description in engineering (for example, of a solenoid) is followed by an approximate device equation for its function, for example, its input-output relation.

No one knows which biological systems can be viewed productively as devices. No one knows how many of the unsolved complexities of biological research reflect problems of the reverse engineering of simple devices, and how many reflect the inherent complexity of biological systems. One can certainly imagine simple systems that are hard to investigate because of the paucity of experimental knowledge. Complex systems - for example, with many internal nonlinear connections like the integrated circuit modules of digital computers or, perhaps, the central nervous system - may not be easily analysed as devices, no matter how many experimental data are available. But it seems clear, at least to a physiologist, that productive research is catalysed by assuming that most biological systems are devices. Thinking today of your biological preparation as a device tells you what experiments to do tomorrow.

Asking the questions in this way leads to the design of useful experiments that may eventually lead to the device description or equation, if it exists. If no device description emerges after extensive investigation of a biological system, one can look for other, more subtle descriptions of nature's machines.

\section{R. S. Eisenberg}

Department of Molecular Biophysics and

Physiology, Rush Medical Center,

1653 West Congress Parkway,

Chicago, Illinois 60612, USA

\section{Endowments are necessary for museums to thrive}

SIR - You suggest that combining science and outreach might help museums succeed in achieving the funding they need for research, in your Editorial 'Museums need two cultures', News story 'Smithsonian looks beyond ousted boss' and News Feature 'Endangered collections' (Nature 446, 583, 594 and 605-606; 2007). As the current and former heads of such institutions, we emphasize that this combination can succeed only if followed up with major fund-raising for endowments.

The slow growth in research funding is being countered with increases in the number of investigators. Moreover, cost recovery from grants never covers full institutional costs, and income from exhibitions barely covers the costs of the exhibitions themselves. Most museums, botanical gardens and related institutions are substantially under-endowed.

Recognizing this situation, the Visiting Committee for Smithsonian Science has called for a major campaign to build a new endowment of at least $\$ 1$ billion. Without such a source of unencumbered funds, research and outreach in such institutions will starve. Institutions that have resisted this kind of fundraising, including the Smithsonian and the Academy of Natural Sciences, will continue to struggle financially.

Adequate endowment is particularly important for institutions with extensive and irreplaceable collections of biological specimens. It would be a tragedy if we did not capitalize on the knowledge we could gain by providing adequate funding for this research. Growing capital wealth in all parts of the world today means that there is a new opportunity to build the endowments for these unique institutions.

D. James Baker ${ }^{\star}$, Jeremy A. Sabloff $\dagger$, Peter A. Raven

*Visiting Committee for Smithsonian

Science, 8031 Seminole Avenue, Philadelphia,

Pennsylvania 19118-3915, USA

†University of Pennsylvania Museum of

Archaeology and Anthropology,

3260 South Street, Philadelphia,

Pennsylvania 19104-6324, USA

†Missouri Botanical Garden, PO Box 299,

St Louis, Missouri 63166-0299

\section{Bright idea to improve prose but remain accurate}

SIR - I agree with Cheryl Strauss

('Increasing prose quality by decreasing repetition' Nature 446, 725; 2007) that it would be pleasing to find trimmer descriptions in the scientific literature. It is tempting to blame overuse of words such as 'increase' and 'decrease' on a linguistically repressive science culture.

Nevertheless, scientific communication aims to record information explicitly, leaving nothing to contextual interpretation, so that experiments may be repeated and verified. Unconventional description has the dual danger of being imprecise or too specific.

Take, for example, the suggestion of replacing 'increased' with 'brighter' or 'more intense' to qualify the word 'fluorescence.' All three expressions have explicit, exclusive definitions. 'Increased' fluorescence means that the number of photons emitted due to absorption of smaller-wavelength photons is larger than it was. If a sample is 'brighter', the luminous flux per unit area per unit solid angle has increased, scaled for human visual receptors. And if a sample is 'more intense', it might be fluorescing at the same rate into a smaller area. A scientist reading the article might make an incorrect assumption if the wrong qualifier is used. We must ensure that editing preserves precise scientific meanings.

I agree, though, that 'shorter mouse tails' is an improvement on 'mouse tails of decreased length' - no one is likely to assume they were shorter in time! Brad Deutsch

Institute of Optics, Wilmot 121,

University of Rochester, River Campus,

Rochester, New York 14611, USA

Contributions to Correspondence may be submitted to correspondence@nature.com. They should be no longer than $\mathbf{5 0 0}$ words, and ideally shorter. They should be signed by no more than three authors; preferably by one. Published contributions are edited. 